\title{
Reconstruction of Object Position in X-ray Mammograms
}

P. Mikeš

Keywords: reconstruction, decompression, mammography.

\section{Introduction}

Female breast cancer is one of the most frequent causes of death in the female population of all age groups today. Many research groups have worked on mammogram computer processing with various degrees of success. Calcifications and their relatively well-known links with various breast disorders are most frequently used for valuation of malignity. These calcifications can be viewed as individual objects or clusters of them (if present) can be investigated. A classical mammographic investigation provides two photos from two different angles, while the breast is being compressed by compress plates. This procedure does not allow us to classify the shape of the calcifications in their three-dimensional appearance, but only as a projection from two different directions. This is frequently adequate to determine whether they are malignant. However, a cluster is a three-dimensional structure in grain and it can only with difficulty be assessed in its original 3D form on the basis of two projections. I will suggest Table 1 Progress of reconstruction and decompression a way to obtain this information more exactly without needing to produce more photos. It means that we can gain more diagnostic insight without modifying the classical investigation. For the purposes of this investigation we will assume a mammogram in its digital form, acquired by a digital mammograph or digitized out of a film.

\section{Recording method}

The situation during recording and subsequent processing is summarized in Table 1. A female breast is held in so-called compress plates before photography (phase 1). There are many sound reasons for this step, and it is used for all mammographic investigations today. However, this step makes it somewhat harder to classify the objects in the breast, especially the calcification clusters and microcalcifications, because mammograms provide a picture of a compressed breast. If we obtain a reconstructed three-dimensional picture of a breast, it is necessary to remember

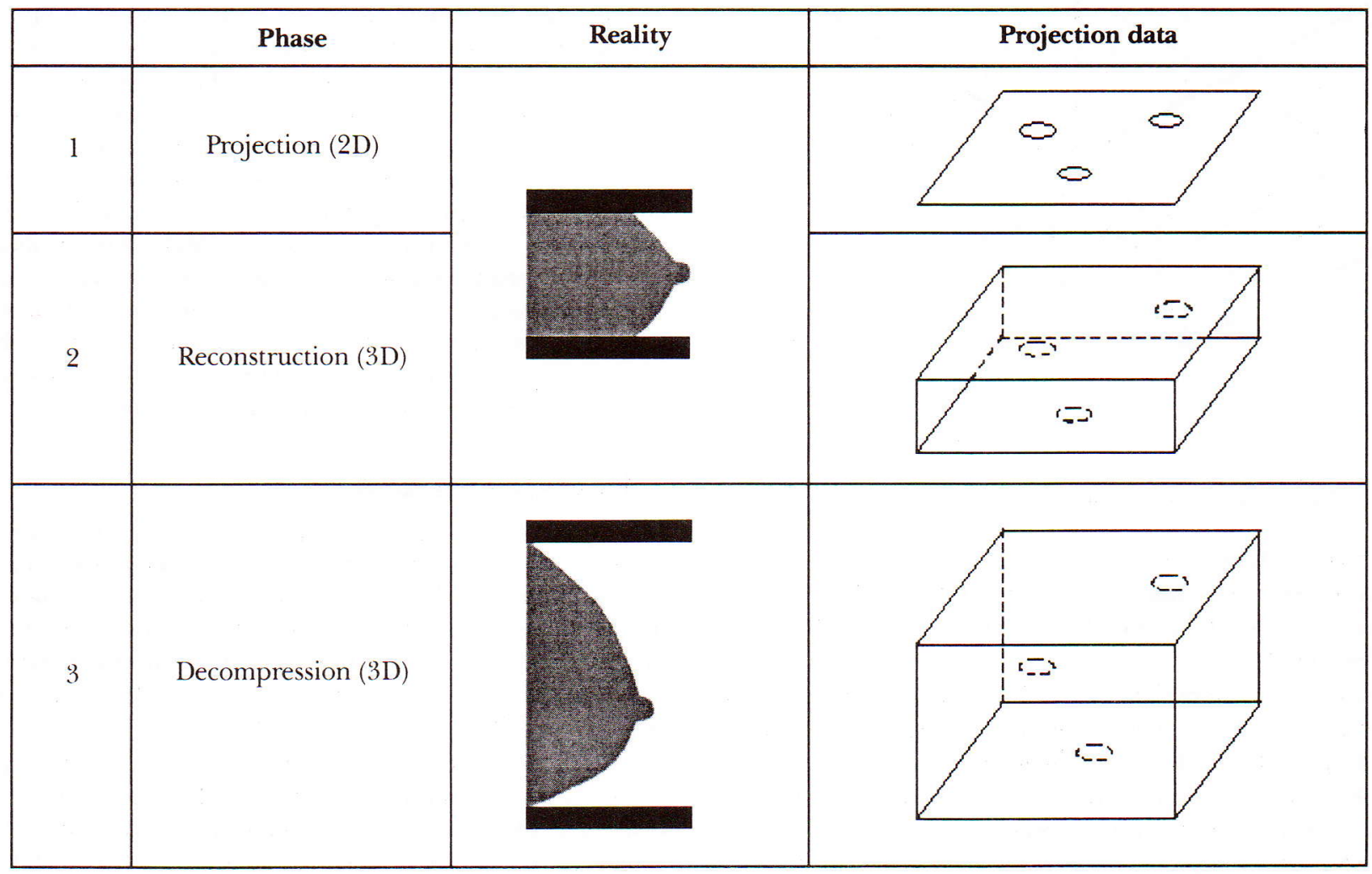


that we are dealing with a compressed breast (phase 2). Thus we have to decompress the three-dimensional structure acquired from mammograms by a reverse process (phase 3 ).

\section{Reconstruction}

For the purposes of reconstruction if is suitable to set up a co-ordinate system reflecting the conditions during photography and during subsequent analysis, see Fig. 1. Let us assume that a breast nipple (M) between compression plates (1) is positioned approximately in a parallel plane half way between the plates (2). This holds only if we allow definite deviation, but on the other hand there is no other better fixed point in this system and we have to accept this presumption and attempt to correct any deficiencies. The position of the nipple ( $\left.\mathrm{M}^{\prime}\right)$ can be determined well in the resultant photos (3). It is therefore appropriate to set the beginning of the co-ordinate system at the center of the nipple. As regards the method for holding the breast in the compress plates, the axis can be led through the center of the breast and through the nipple upright to the plane of the body. This axis will be pointed away from the nipple toward the body - and in this way the orientation of the $y$-axis can be defined. Then we can have the $z$-axis vertical from the nipple to the half-space in which the X-ray tube is focused (F). The $x$-axis then logically fulfills a clockwise coordinate system.

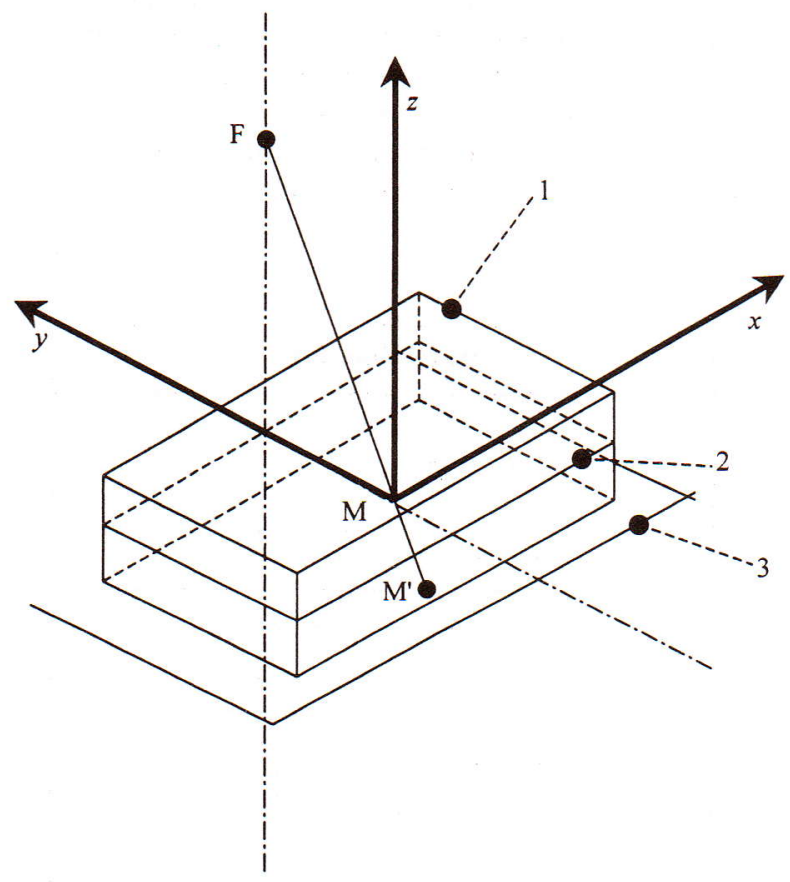

Fig. 1: Definition of the co-ordinate system

Have in mind that:

1. at every investigation the plane parallel to the compression plates and passing through the nipple is not affected by compression,

2. since one dimension of the breast always remains uncompressed, the axis of the breast passing through the nipple is never deformed during compression,

3. thus it is appropriate to place one of the coordinates of the coordinate system into this breast axis, which will be used for determining the positions of all objects.
As a result, the whole mammograph system can be regarded as a system rotating equally around the $y$-axis under given conditions. Thus, two projections will be adequate for a $3 \mathrm{D}$ reconstruction of the positions of the entities displayed in the photographs.

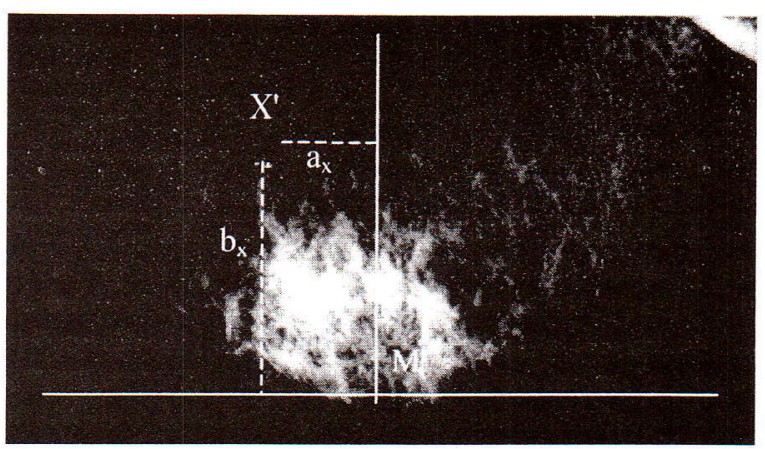

Fig. 2: Position determination in a real photo

The next step is to uniquely determine a given object in both pictures (Fig. 2). When this has been done, the next steps involve the application of analytical geometry:

1. place the $y$-axis through the axis of the breast,

2. put the beginning of the coordinate system in the center of the nipple projection ( $\left.\mathrm{M}^{\prime}\right)$,

3. obtain the orientation of the $\mathrm{x}$-axis and of the $\mathrm{z}$-axis,

4. find the real point $X$ projection (on picture $X^{\prime}$ ),

5. determine the object coordinates in both scans $\left(a_{x}, b_{x}\right)$.

In this way, the acquired data, together with the X-ray tube focus position coordinates (as is well known from service documentation) will be sufficient to provide an analytical representation by two straight lines that always pass through the focus and through one of two projections of the object. In the best case the straight lines will cross at a single point. In practice, this is not what happens. We will therefore look for the point coordinate that is placed at the point where these straight lines come closest together. The smallest distance of two skews can be determined by retrieving their axis. Two planes can later define this axis. Now we will find the point of intersection of these two planes with the first straight line to obtain point I and with the second straight line to obtain point J. Our searched point X is obtained simply as the center of the abscissa given by points I and J. The length of this abscissa may be a measure of the reconstruction accuracy, because in the best case the two straight projection lines would have to intersect and points X, I and J would merge.

\section{Decompression}

Above, we determined the spatial location of the objects. However, we have been defining the distribution of objects in a breast compressed by compression plates. Now we have to deal with "decompression" of the breast, in order to obtain the real position of the points in the breast. There are approximately 4 models of a situation in a breast after it has been compressed. These are shown in Table 2.

In the literature, models $\mathrm{C}$ and $\mathrm{D}$ receive most attention. We make the following assumptions. The compressed fat tissue will shift and the breast mass will redistribute. Any compression or shape change has no influence on the analysis 
Table 2: Models of compressed breast behavior

\begin{tabular}{|c|c|c|c|c|}
\hline \multirow{2}{*}{ model } & \multicolumn{2}{|c|}{ fat tissue } & \multicolumn{2}{c|}{ tissue of interest } \\
\cline { 2 - 5 } & compression & shift & compression & shift \\
\hline A & yes & yes & no & no \\
\hline B & yes & yes & no & yes \\
\hline C & yes & yes & yes & no \\
\hline D & yes & yes & yes & yes \\
\hline
\end{tabular}

process, because the microcalcifications of interest (i.e., those leading to malignant cases) are obviously not present in this tissue type. A milky gland is compressed as a structure in the environment of a fat tissue. With respect to the character of the structure, the shape changes can be considered as homogeneous body compressions. The specific "branches" may also shrink under compression, but not significantly, and we again assume that there is only a homogeneous body compression. As regards the structure, the error in the thickness of the branches is inconsiderable thanks to the presupposition of homogeneity. This means that model $\mathrm{D}$ is correct, but it has a small compression and will come up to model B. If this presumption is valid, then the decompression is relatively easy, but the parameters for a mathematical model of the decompression will have to be obtained from practical experiments. It is necessary to take into account the structure of a breast, especially the gland-to-fat tissue ratio, which is very variable, and depends on age. On the other hand, membership of a given category can be estimated from the mammogram. For the purposes of reconstruction, is possible to continue with admission of these tolerances. Further research will aim at making a more precise definition.

Experimental verification will make use of the similarity between human female breast tissue structure and that of a sow's breast. The following verification progress is suggested:

1. obtain a sow's breast,

2. insert contrastive non-metallic markings into the tissue using a hollow needle in order to overlay the whole test tissue. The motion of the marks in both tissue types will be studied separately, to avoid the need to distribute them equally,

3. verify the specific positions of these objects using computer tomography, using a tool that has already been fabricated to replace compress plates,

4. perform several compression steps, and investigate the position of the signs, using a tomograph,

5. perform a classical mammographic investigation,

6. analyze this result and compare it with the results from CT.
We will then be able to document the accuracy of the reconstruction algorithm and obtain information about tissue behavior during compression (point 4).

\section{References}

[1] Dhawan, A. P., Chitre, Y., Kaiser-Bonasso, C., Moskowitz, M.: Analysis of Mammographic Microcalcifications Using Gray-Level Image Structure Features. IEEE Trans. on Medical Imaging, Vol. 15, 1996, No. 3, p. 246-259.

[2] Dengler, J., Behrens, S., Desaga, J. F.: Segmentation of Microcalcification in Mammograms. IEEE Trans. on Medical Imaging, Vol. 12, 1993, No. 4, p. 634-642.

[3] Bowyer, K. W., Astley, S.: State of Art in Digital Mammographic Image Analysis. Salem: World Scientific Publishing Co. Pte. Ltd., 1994, p. 291.

[4] Kilday, J., Palmieri, F., Fox, M. D.: Classifying Mammographic Lesions Using Computerized Image Analysis. IEEE Trans. on Medical Imaging, Vol. 12, 1993, No. 4, p. 664-669.

[5] Jain, A. K.: Fundamentals of Digital Image Processing. New Jersey: Prentice-Hall International, Inc., 1989, p. 569.

[6] Highnam, R., Brady, M.: Mammographic Image Analysis. Dordrecht: Kluwer Academic Publishers, 1999, p. 379.

[7] Sadjadi, F. A., Hall, E. L.: Three-dimensional moment invariants. IEEE Trans. on Pattern Analysis and Machine Intelligence. Vol. 2, March 1980, No. 2, p. 127-136.

[8] Mikeš, P.: Detekce a klasifikace mikrokalcifikaci v rentgenových mammogramech. In: Moderní mikroskopie. Brno: Česká společnost pro vědeckou kinematografii. 1998, p. 93-102.

[9] Mikeš, P.: Detection E Classification of X-Ray Mammogram Calcifications. In: Biosignal 2000 Proceedings. Brno: TU, 2000, p. 275-277.

$$
\begin{aligned}
& \text { Ing. Petr Mikeš } \\
& \text { phone: } \\
& \text { +420234052417 } \\
& \text { +420605748927 } \\
& \text { e-mail: mikes@ttc.cz }
\end{aligned}
$$

Development Department,

TTC Telekomunikace,

Třebohostická 5

143 00, Prague 10, Czech Republic

Institute of Radioelectronics

Czech Technical University in Prague

Technická 2

166 27, Prague 6, Czech Republic 